\title{
Prognostic Factors in Patients with Septic
} Disseminated Intravascular Coagulation Treated with Thrombomodulin: The Effect of Reduced Thrombomodulin Dose; a Single-center, Retrospective, Observational Study

Yoshihiro Nishita ( $\Delta$ yoshi-n@kanazawa-med.ac.jp)

Kanazawa Medical University Hospital

Masatoshi Taga

Kanazawa Medical University Hospital

Masaru Sakurai

Kanazawa Medical University

Yoshitsugu linuma

Kanazawa Medical University

Togen Masauji

Kanazawa Medical University Hospital

\section{Research Article}

Keywords: Human soluble thrombomodulin alfa, Sepsis, Disseminated intravascular coagulation (DIC), dose reduction, DIC resolution

Posted Date: November 1st, 2021

DOI: https://doi.org/10.21203/rs.3.rs-1027874/v1

License: (c) (i) This work is licensed under a Creative Commons Attribution 4.0 International License.

Read Full License 


\section{Abstract}

Background: Human soluble recombinant thrombomodulin (TM alfa), a treatment for septic Disseminated intravascular coagulation (DIC), is recommended for patients with severe renal dysfunction in reduced doses. However, no studies have examined yet how dose reduction affects clinical efficacy. In this study, we investigated the significance of the TM alfa dose as a prognostic factor in clarifying the clinical background factors related to the clinical effect of TM alfa in patients with septic DIC.

Methods: This study involved 102 patients with septic DIC admitted to a single-center intensive care unit between April 2013 and March 2020, receiving TM alfa. The following factors were retrospectively collected from the medical records of the target patients: (1) patient background, (2) sequential organ failure assessment (SOFA) score, (3) Japanese Association for Acute Medicine DIC diagnostic criteria score, (4) DIC treatment information, (5) TM alfa dose per bodyweight (normal dose: $0.06 \mathrm{mg} / \mathrm{kg}$ or reduced dose: $0.02 \mathrm{mg} / \mathrm{kg}$ ), (6) DIC resolution within 7 days after the start of TM alfa administration (DIC resolution), (7) all deaths within 30 days after the start of TM alfa administration (30-days-all-cause mortality), (8) presence or absence of new hemorrhagic side effects after the start of TM alfa administration. Multiple logistic regression analysis was used to assess factors associated with DIC resolution and 30-days-all-cause mortality.

Results: The SOFA score (odds ratio: $95 \%$ confidence interval, $0.76: 0.66-0.89)$, pneumonia (0.24: 0.08$0.75)$, and reduced dose administration of TM alfa (0.23: 0.08-0.66) were independent of and negatively related to the DIC resolution. For the 30-days-all-cause mortality, the SOFA score (1.66: 1.31-2.09), pneumonia (9.50: 2.49-36.25), and TM alfa dose reduction (3.52: 1.06-11.69) were independent, poor prognostic factors. We found no association between the hemorrhagic side effects and the TM alfa dose per bodyweight.

Conclusions: The reduced dose of TM alfa for patients with severe renal dysfunction was observed to be an influential factor for DIC resolution and 30-day all-cause mortality in addition to SOFA score and pneumonia. Further studies are required in the future to verify this finding.

\section{Background}

Sepsis is a major cause of intensive care unit (ICU) admission in the developed countries, including Japan, and is a condition with a high mortality rate [1,2]. The mortality rate is reportedly higher in patients with disseminated intravascular coagulation (DIC) [3-5], although the treatment of patients with DIC has yet not been properly established.

Thrombomodulin (TM) alfa, a recombinant human soluble thrombomodulin, is an anticoagulant drug approved and introduced in Japan in 2008 for the treatment of DIC [6]. Like thrombomodulin, TM alfa not only directly inhibits the coagulation activity of thrombin by forming a complex with it but also helps to activate protein $\mathrm{C}$, an anticoagulant factor, and to inactivate activated factor $\mathrm{V}$ and factor VIII, thereby inhibiting blood coagulation [6,7]. In addition, TM alfa is frequently used for sepsis-associated DIC due to 
its lectin-like domain-mediated anti-inflammatory effects, recent meta-analyses proving its clinical efficacy $[8,9]$. Therefore, the Surviving Sepsis Campaign Guidelines [10] and the Japanese Sepsis Guidelines 2016 did not provide a clear recommendation for TM alfa in patients with septic DIC, although the latest Japanese Sepsis Guideline 2020 weakly recommends TM alfa in patients with septic DIC.

The urinary excretion rate of TM alfa within 48 hours after administration has been reported to be $54.3 \%$ $59.8 \%$ [11]. TM alfa is usually used at a dose of $0.06 \mathrm{mg} / \mathrm{kg}(380 \mathrm{U} / \mathrm{kg})$ but in patients with severe renal dysfunction, the dose could be reduced to $0.02 \mathrm{mg} / \mathrm{kg}(130 \mathrm{U} / \mathrm{kg})$ according to the symptoms. Nevertheless, Hayakawa et al. reported no difference in the TM alfa blood concentration depending on the dosage between patients with Creatinine clearance (CCr) less than $10 \mathrm{~mL} / \mathrm{min}$ and those with $\mathrm{CCr}$ greater than $10 \mathrm{~mL} / \mathrm{min}$ [12]. Therefore, the effective blood concentration might not be reached at a dose of $0.02 \mathrm{mg} / \mathrm{kg}$ of TM alfa in patients with renal dysfunction. Imaura et al. reported a significantly higher 90-day survival rate in patients with plasma levels of TM alfa higher than $600 \mathrm{ng} / \mathrm{mL}$ [13]. Therefore, reaching an effective TM alfa plasma concentration seems to be important for improving clinical outcomes. However, no currently available reports have examined how the different doses ( 0.06 or 0.02 $\mathrm{mg} / \mathrm{kg}$ ) could affect clinical efficacy in patients with severe renal dysfunction.

In this study, we aimed at clarifying the clinical background factors associated with the prognosis of patients with septic DIC treated with TM alfa, such as DIC resolution, death, and the occurrence of hemorrhagic complications. Based on the results of these analyses, we discussed the significance of the TM alfa dose as a prognostic factor.

\section{Methods}

This was a single-center, retrospective study of adult patients with septic DIC treated with TM alfa in the ICU of Kanazawa Medical University Hospital, approved by the Ethical Review Committee of Kanazawa Medical University Hospital (Approval number: H263). Due to the retrospective nature of the study, informed consent was waived.

\section{Patients}

This study was conducted between April 1, 2013 and March 31, 2020, including patients admitted to the mixed medical ICU and surgical ICU of Kanazawa Medical University Hospital, with a total of 18 beds. We included in the study those patients who received TM alfa and fulfilled the following criteria: adult patients aged at least 20 years, selected using the Sepsis-3 diagnostic criteria (suspected infection and a sudden increase in Sequential Organ Failure Assessment (SOFA) score of more than 2 points), and the Japanese Association for Acute Medicine DIC diagnostic criteria (JAAM-DIC) score of at least 4 points. We established two dosage groups for TM alfa: (1) $0.06 \mathrm{mg} / \mathrm{kg}$ with or without severe renal dysfunction [CCr $<15 \mathrm{~mL} / \mathrm{min}$ or oliguria (urine output $<0.5 \mathrm{~mL} / \mathrm{kg} / \mathrm{hr}$ )] (normal dose group) and (2) $0.02 \mathrm{mg} / \mathrm{kg}$ for severe renal dysfunction (reduced dose group). However, doses set at $\pm 5 \mathrm{~kg}$ bodyweight were included in the analysis of this study. The following patients were excluded: (1) patients who did not meet the criteria 
for JAAM-DIC diagnosis immediately before TM alfa administration, (2) patients younger than 20 years, (3) patients receiving a dose set different from at $0.02 \mathrm{mg} / \mathrm{kg}$ or $0.06 \mathrm{mg} / \mathrm{kg}$, (4) patients with no decline in renal function $(\mathrm{CCr} \geq 15 \mathrm{~mL} / \mathrm{min}$ and urine output $\geq 0.5 \mathrm{~mL} / \mathrm{kg} / \mathrm{hr}$ ) and with a dose set $0.02 \mathrm{mg} / \mathrm{kg}$, (5) patients with a TM alfa dose changed during the DIC treatment, (6) hematology patients with a platelet count consistently less than $80,000 / \mu \mathrm{L},(7)$ patients with a treatment period shorter than 2 days.

\section{Variables and outcomes}

The following immediately-before-TM alfa-administration factors were investigated retrospectively from the medical records: patient background; age, sex, height, weight, $\mathrm{CCr}$, presence of severe renal dysfunction, blood urea nitrogen, presence of renal replacement therapy, presence of malignancy, serum albumin, antithrombin III (AT III), C reaction protein, type of infection, SOFA score, JAAM-DIC score, systemic inflammatory response syndrome (SIRS) criteria. The following factors were investigated as parts of the DIC treatment: antimicrobial agents, AT III, fresh frozen plasma (FFP), platelet concentrate (PC), sodium heparin (except for low-dose sodium heparin for deep vein thrombosis prophylaxis and saline with heparin for line keeping), TM alfa dose per bodyweight ( 0.06 or $0.02 \mathrm{mg} / \mathrm{kg}$ ), and Polymyxin B immobilized fiber column direct hemoperfusion (PMX-DHP). The following outcome factors were investigated: DIC resolution within 7 days of TM alfa initiation, 30-days-all-cause mortality from TM alfa initiation, and the presence and details of any new bleeding side effects after TM alfa initiation (bleeding site, whether the bleeding was major bleeding according to the International Society of Thrombosis and Hemostasis bleeding criteria) [14]. The resolution of DIC was defined as a JAAM-DIC score of fewer than 4 points.

\section{Statistical analysis}

We exploratively examined the clinical factors associated with each outcome: DIC resolution within 7 days of starting TM alfa, 30-days-all-cause mortality from TM alfa initiation, and new bleeding complications after TM alfa initiation. The baseline attributes and treatment methods were compared between the patients with and without each outcome.

The data are presented as the median (interquartile range) or number (\%). The chi-square test or Fisher's exact test were performed for univariate analysis of categorical variables, and the Mann-Whitney Utest was performed for univariate analysis of the continuous variables. Factors associated with DIC resolution within 7 days of TM alfa initiation and 30-days-all-cause mortality were evaluated using multiple logistic regression analysis. The objective variable was the occurrence of each outcome. For the explanatory variables, all baseline subject attributes associated with the outcome $(p<0.1)$ were entered into the model, and the relevant variables were selected using the stepwise (increasing variable) method. Confidence interval $(\mathrm{Cl})$ was calculated. For the outcome of hemorrhagic complications, the relevant background factors were evaluated by univariate analysis but the multivariate analysis was not performed for hemorrhagic complications due to the small number of cases. 
We used the SPSS software version 27 (IBM Corp, Armonk, New York, USA) for statistical analysis, and two-sided P-values of $p<0.05$ were considered statistically significant.

\section{Results}

A total of 102 patients who received TM alfa during the study period were included in the study.

Table 1 shows the patient background. The median age (interquartile range) was 76 years (67-83), and 58 patients (56.9\%) were male. This study included 54 patients (52.9\%) with severe renal dysfunction. Of these, 30 (29.4\%) received reduced doses, and 72 (70.6\%), including 48 non-decreased renal function patients, normal doses. Renal replacement therapy was performed in 55 patients (53.9\%). The median (interquartile range) SOFA score was 11 (9-14), the median (interquartile range) JAAM-DIC score was 6 (5-7), and the median (interquartile range) SIRS criterion was 3 (2-4). Twenty-five (24.5\%) patients had intra-abdominal infection, 23 (22.5\%) had pneumonia, and 17 (16.7\%) had urinary tract infection. Thirty (29.4\%) patients received reduced doses of TM alfa with a median treatment duration of 6 days (4-6). Antimicrobial agents were administered to all patients. 78 patients (76.5\%) received AT III, 3 patients $(2.9 \%)$ received heparin sodium, 29 patients (28.4\%) received FFP, and 37 patients (36.3\%) received PC. PMX-DHP was performed in 48 patients (47.1\%). The clinical outcomes were 53.9\% (55/102) of patients with DIC resolution within 7 days, 29.4\% (30/102) with 30-days-all-cause mortality, and 12.7\% (13/102) with bleeding complications.

Table 2 shows the relationship between the DIC resolution and clinical background. Compared with the DIC resolution group, the non-DIC resolution group contained significantly more males, just as well as patients with renal replacement therapy and significantly lowers CCr. The SOFA score was significantly higher in the non-DIC resolution group. Pneumonia was more common in the non-DIC resolution group, while cholangitis/cholecystitis was significantly more common in the DIC resolution group. A reduced TM alfa dose was more common in the non-DIC resolution group, and FFP was significantly less common in the non-DIC resolution group. The JAAM-DIC score, unknown type of infection, and PC administration tended to be associated with DIC resolution $(p<0.1)$. All the factors associated with the presence or absence of DIC resolution were entered into the model, and the factors associated with the outcome were selected by the logistic regression analysis stepwise method. As a result, three factors were selected: SOFA score, pneumonia, and reduced TM alfa dosage. These were found to be significantly associated with DIC resolution independently of each other (Table 3). That is, the higher the SOFA score, the lower the odds ratio (OR) for the DIC resolution (OR for DIC resolution per 1 increase in SOFA score, 0.76; $95 \% \mathrm{Cl}$, 0.66-0.89). Pneumonia had a significantly lower OR for the DIC resolution (OR, 0.24; 95\% $\mathrm{Cl}, 0.08-0.75)$, and the TM OR for the DIC resolution was significantly lower in the TM alfa reduced dose group compared with the normal dose group (OR, 0.23; 95\% $\mathrm{Cl}, 0.08-0.66)$.

Table 4 shows the relationship between the presence of the 30-days-all-cause mortality and the clinical background. The SOFA score was significantly higher, pneumonia and TM alfa dose reduction were significantly more common, and PMX-DHP was significantly less common in the death group. Serum 
albumin and cholangitis/cholecystitis tended to be associated with the 30-days-all-cause mortality ( $p<$ 0.1 ). The 30-days-all-cause mortality was defined as an outcome, and the related factors were evaluated by logistic regression analysis. The results showed that a high SOFA score (OR, 1.66; 95\% $\mathrm{Cl}, 1.31-2.09)$, pneumonia (OR, 9.50; 95\% Cl, 2.49-36.25), and reduced TM alfa dose (OR, 3.52; 95\% Cl, 1.06-11.69) were independently associated with a significant increase in the OR (Table 5).

Bleeding adverse reactions were observed in 13 patients (12.7\%) (Table 6). The major sites of bleeding were of subcutaneous, oral, and gastrointestinal localization in three cases each, followed by bleeding from the site of vascular penetration in two cases. One major bleeding case of gastrointestinal bleeding was observed in a patient with reduced dosage on TM alfa. As for the factors associated with hemorrhagic adverse reactions (Table 7), infectious enteritis was significantly more common but no differences in other factors have been observed.

\section{Discussion}

Although septic DIC is a disease with a poor prognosis [3-5], few drugs have been proven clinically effective for its treatment. TM alfa is weakly recommended in the Japanese guideline for sepsis 2020, and several reports have been published on the efficacy of TM alfa in septic DIC [15-18]. Furthermore, a recent meta-analysis has shown the benefit of TM alfa in septic DIC resolution and 28-days-all-cause mortality [8-9]. Therefore, TM alfa has become one of the options for septic DIC. However, some of the reports in the meta-analysis excluded patients with severe renal dysfunction [19-21], and the efficacy of $\mathrm{TM}$ alfa at a reduced dose of $0.02 \mathrm{mg} / \mathrm{kg}$ has not been properly addressed either. Therefore, in this study, we focused on the TM alfa dose and examined how it could affect clinical efficacy in patients with septic DIC.

In this study, a reduced TM alfa dose was identified as a factor associated with the resolution of septic DIC and 30-days-all-cause mortality. These results indicate that TM alfa should be administered at a dose of $0.06 \mathrm{mg} / \mathrm{kg}$ regardless of renal function to maintain effective plasma concentrations and achieve full efficacy. In recent years, certain studies reported on the relationship between TM alfa pharmacokinetics and its clinical effects. For example, TM alfa plasma concentrations were reportedly similar in patients with different renal functions ( $\mathrm{CCr}<10 \mathrm{~mL} / \mathrm{min}$ vs $10 \mathrm{~mL} / \mathrm{min} \leq \mathrm{CCr}<30 \mathrm{~mL} / \mathrm{min}$ vs $30 \mathrm{~mL} / \mathrm{min} \leq \mathrm{CCr}<$ $60 \mathrm{~mL} / \mathrm{min}$ vs $60 \mathrm{~mL} / \mathrm{min} \leq \mathrm{CCr}$ ) [12]. In addition, TM alfa is a renal excretory drug, and the 24-hour urinary excretion rate correlates with $\mathrm{CCr}$. Moreover, it has been pointed out that other metabolic processes, such as activated elastase-mediated degradation, might have a compensatory effect on the disappearance of TM alfa in patients with DIC and renal dysfunction [22,23]. Another study on a case of septic DIC with a TM alfa plasma concentration below $600 \mathrm{ng} / \mathrm{mL}$ reported that the decrease in the JAAM-DIC score from the baseline 4 days after TM alfa administration and the 90-day survival rate were significantly higher and lower, respectively [13]. Therefore, the TM alfa dosage in patients with severe renal dysfunction might be important in improving the prognosis of patients with septic DIC. 
In this study, the SOFA score was a proven independent factor associated with the resolution of DIC and 30-days-all-cause mortality. The SOFA score has been adopted as a diagnostic criterion for sepsis as an indicator of organ damage severity [24]. In a previous study, the SOFA score was reportedly associated with the 28-day mortality in patients with DIC [5]. Therefore, organ damage severity in patients with DIC could be related to prognosis. In addition, when patients with DIC were classified by the SOFA score, TM alfa reportedly improved the survival in a high-risk subset with a SOFA score of 13-17 [19]. In addition to the high risk of death in patients with a high SOFA score, TM alfa might improve clinical outcomes. Therefore, the TM alfa dosage should be determined more carefully.

In this study, the most common sites of infection were the abdomen, lung, and urinary tract, respectively. Interestingly, in the Japanese Sepsis Registry study, the most common sites of infection causing sepsis were the lung, abdomen, and urinary tract, respectively [3]. In a study of Japanese ICUs, the most common sites of infection causing sepsis were the lung, abdormen, and urinary tract, respectively [1]. In an international study, respiratory, intra-abdominal, and bloodstream infections were the most common infectious sites in the ICU [25]. In the present study, intra-abdominal infection was the most common, although the infected sites were similar to those in the previous studies of sepsis. This might be due to the fact that intra-abdominal infections have a higher risk of developing into DIC [26] since they are often subjected to stress due to surgery in addition to organ damage caused by infection. In the present study, pneumonia was associated with DIC resolution and 30-day all-cause mortality, indicating that pneumonia-associated DIC might be an independent factor in mortality regardless of the TM alfa dose. In the case of sepsis, pneumonia reportedly exhibited a higher in-hospital mortality rate than in the case of intra-abdominal and urinary tract infections [3]. Moreover, a retrospective Japanese nationwide study [27] reported that TM alfa did not improve the 28-day mortality in pneumonia-associated DIC. The usefulness of TM alfa in the treatment of pneumonia-associated DIC needs to be further investigated.

In this study, the incidence of major bleeding was $1.0 \%$ (only one case of bleeding occurred in a patient with reduced dosage), and the overall incidence of bleeding was $12.7 \%$. The background factors related to hemorrhagic complications were investigated but due to the insufficient number of patients, hemorrhage-associated factors could not be identified. In the previous TM alfa randomized controlled trials, no significant difference could be observed in the incidence of major bleeding compared with the placebo control $[19,20]$. In patients with renal dysfunction, the TM alfa blood concentration reportedly did not increase excessively even at normal doses due to the effect of other compensatory metabolic processes such as activated elastase-mediated degradation [12,22,23]. The TM alfa dose is not expected to be associated with hemorrhagic complications, although further studies would be required in this field.

No previous studies have reported how TM alfa, including its dosage, could affect clinical outcomes. In this study, we showed for the first time that the normal TM alfa dose in patients with severe renal dysfunction might be a factor that improves short-term clinical outcomes without increasing adverse bleeding events. However, this study also has several limitations. First, this was a single-center, retrospective study, and the influence of potential confounders cannot be ruled out. 
Second, this study examined DIC resolution and 30-days-all-cause mortality but did not examine the longterm prognosis. In a retrospective study, it is difficult to determine the long-term prognosis in multiple cases, a prospective multicenter study would thus be recommended. Third, the present study did not sufficiently examine bleeding complications. TM alfa safety in patients with severe renal dysfunction has not been clarified, although it would be expected that a normal dose of TM alfa would not cause an excessive concentration increase. The relationship between TM alfa dose and hemorrhagic complications in patients with severe renal dysfunction needs further investigation.

\section{Conclusions}

In this study, we showed that a reduced dose of TM alfa in patients with severe renal dysfunction was an influential factor in the SOFA score, pneumonia, DIC resolution, and 30-days-all-cause mortality. A prospective, multicenter interventional study is warranted to validate this finding.

\section{Abbreviations}

ICU: Intensive care unit; DIC: Disseminated intravascular coagulation; TM: Thrombomodulin; CCr: Creatinine clearance; SOFA: Sequential organ failure assessment; JAAM-DIC: Japanese Association for Acute Medicine DIC diagnostic criteria; AT囚: Antithrombin 『; CRP: C reaction protein; SIRS: Systemic inflammatory response syndrome; FFP: Fresh frozen plasma; PC: Platelet concentrate; PMX-DHP: Polymyxin B immobilized fiber column direct hemoperfusion; OR: Odds ratio; Cl: Confidence interval

\section{Declarations}

\section{Ethics approval and consent to participate}

The study protocol was approved by The Kanazawa Medical University Hospital Institutional Review Board (H263) and optout was done.

\section{Consent for publication}

Not applicable

\section{Availability of data and materials}

The datasets generated and analyzed during the current study are not publicly available due to privacy concerns and institutional policy but are available from the corresponding author on reasonable request.

\section{Competing interests}


The authors declare no competing interests.

\section{Funding}

This study was funded only by the affiliated institutions of the authors.

\section{Authors' contributions}

YN has full access to all study data and takes responsibility for its integrity. All authors contributed to the study conception and design. Data analysis and interpretation: YN, MT, and MS. Manuscript drafting: YN. All authors read and approved the final manuscript.

\section{Acknowledgements}

The authors wish to thank to Clinical Research Support Office in Kanazawa Medical University.

\section{References}

1. Abe T, Ogura H, Shiraishi A, Kushimoto S, Saitoh D, Fujishima S, et al. Characteristics, management, and in-hospital mortality among patients with severe sepsis in intensive care units in Japan: the FORECAST study. Crit Care. 2018;22:322.

2. Prescott HC, Osterholzer JJ, Langa KM, Angus DC, Iwashyna TJ. Late mortality after sepsis: propensity matched cohort study. BMJ. 2016;353:i2375.

3. Ogura H, Gando S, Saitoh D, Takeyama N, Kushimoto S, Fujishima S, et al. Epidemiology of severe sepsis in Japanese intensive care units: a prospective multicenter study. J Infect Chemother. 2014;20:157-62.

4. Lyons PG, Micek ST, Hampton N, Kollef MH. Sepsis-associated coagulopathy severity predicts hospital mortality. Crit Care Med. 2018;46:736-42.

5. Iba T, Di Nisio M, Levy JH, Kitamura N, Thachil J. New criteria for sepsis induced coagulopathy (SIC) following the revised sepsis definition: a retrospective analysis of a nationwide survey. BMJ Open. 2017;7:e017046.

6. Saito H, Maruyama I, Shimazaki S, Aikawa N, Ohno R, Hirayama A, et al. Efficacy and safety of recombinant human soluble thrombomodulin (ART-123) in disseminated intravascular coagulation: results of a phase III, randomized, double-blind clinical trial. J Thromb Haemost. 2007;5:31-41.

7. Wada H, Okamoto K, Iba T, Kushimoto S, Kawasugi K, Gando S, et al. Addition of recommendations for the use of recombinant human thrombomodulin to the "Expert consensus for the treatment of disseminated intravascular coagulation in Japan." Thromb Res. 2014;134:924-5. 
8. Yamakawa K, Levy JH, Iba T. Recombinant human soluble thrombomodulin in patients with sepsisassociated coagulopathy (SCARLET): an updated meta-analysis. Crit Care. 2019;23:302.

9. Yamakawa K, Murao S, Aihara M. Recombinant human soluble thrombomodulin in sepsis-induced coagulopathy: an updated systematic review and meta-analysis. Thromb Haemost. 2019;119:56-65.

10. Rhodes A, Evans LE, Alhazzani W, Levy MM, Antonelli M, Ferrer R, et al. Surviving sepsis campaign: International Guidelines for Management of Sepsis and Septic Shock: 2016. Intensive Care Med. 2017;43:304-77.

11. Nakashima M, Kanamaru M, Umemura K, Tsuruta K. Pharmacokinetics and safety of a novel recombinant soluble human thrombomodulin, ART-123, in healthy male volunteers. J Clin Pharmacol. 1998;38:40-4.

12. Hayakawa M, Kushimoto S, Watanabe E, Goto K, Suzuki Y, Kotani T, et al. Pharmacokinetics of recombinant human soluble thrombomodulin in disseminated intravascular coagulation patients with acute renal dysfunction. Thromb Haemost. 2017;117:851-9.

13. Imaura M, Tsumori M, Nagase S, Omura K, Takahashi H, Hatoyama-Tanaka S, et al. Therapeutic and adverse effects of thrombomodulin alfa to treat sepsis-induced disseminated intravascular coagulation. Shock. 2020;54:50-5.

14. Schulman S, Kearon C. Subcommittee on control of anticoagulation of the Scientific and Standardization Committee of the International Society on Thrombosis and Haemostasis. Definition of major bleeding in clinical investigations of antihemostatic medicinal products in non-surgical patients. J Thromb Haemost. 2005;3:692-4.

15. Mochizuki K, Mori K, Kamijo H, Ichikawa M, Nitta K, Imamura H. Beneficial effect modification on survival outcome of sepsis between ART-123 and polymyxin B-immobilised haemoperfusion: a nationwide Japanese registry study. Ann Intensive Care. 2020;10:57.

16. Hayakawa M, Yamakawa K, Saito S, Uchino S, Kudo D, lizuka Y, et al. Recombinant human soluble thrombomodulin and mortality in sepsis-induced disseminated intravascular coagulation. A multicentre retrospective study. Thromb Haemost. 2016;115:1157-66.

17. Hagiwara A, Tanaka N, Uemura T, Matsuda W, Kimura A. Can recombinant human thrombomodulin increase survival among patients with severe septic-induced disseminated intravascular coagulation a single-centre, open-label, randomised controlled trial. BMJ Open. 2016;6(12):e012850.

18. Hayakawa M, Ono K. A summary of the Japan septic disseminated intravascular coagulation study. Acute Med Surg. 2018;5:123-8.

19. Vincent JL, Francois B, Zabolotskikh I, Daga MK, Lascarrou JB, Kirov MY, et al. Effect of a recombinant human soluble thrombomodulin on mortality in patients with sepsis-associated coagulopathy: the SCARLET randomized clinical trial. JAMA. 2019;321:1993-2002.

20. Vincent JL, Ramesh MK, Ernest D, LaRosa SP, Pachl J, Aikawa N, et al. A randomized, double-blind, placebo-controlled, Phase $2 \mathrm{~b}$ study to evaluate the safety and efficacy of recombinant human soluble thrombomodulin, ART-123, in patients with sepsis and suspected disseminated intravascular coagulation. Crit Care Med. 2013;41:2069-79. 
21. Aikawa N, Shimazaki S, Yamamoto Y, Saito H, Maruyama I, Ohno R, et al. Thrombomodulin alfa in the treatment of infectious patients complicated by disseminated intravascular coagulation: subanalysis from the phase 3 trial. Shock. 2011;35:349-54.

22. Boehme, MW, Galle P, Stremmel W. Kinetics of thrombomodulin release and endothelial cell injury by neutrophil-derived proteases and oxygen radicals. Immunology. 2002;107:340-9.

23. Gando S, Kameue T, Matsuda N, Hayakawa M, Hoshino H, Kato H. Serial changes in neutrophilendothelial activation markers during the course of sepsis associated with disseminated intravascular coagulation. Thromb Res. 2005;116:91-100.

24. Singer M, Deutschman CS, Seymour CW, Shankar-Hari M, Annane D, Bauer M, et al. The third International Consensus definitions for sepsis and septic shock (Sepsis-3). JAMA. 2016;315:801-10.

25. Vincent JL, Rello J, Marshall J, Silva E, Anzueto A, Martin CD, et al. International study of the prevalence and outcomes of infection in intensive care units. JAMA. 2009;302:2323-9.

26. Okamoto K, Tamura T, Sawatsubashi Y. Sepsis and disseminated intravascular coagulation. J Intensive Care. 2016;4:23.

27. $T, H, H, K, H$. Recombinant human soluble thrombomodulin and mortality in severe pneumonia patients with sepsis-associated disseminated intravascular coagulation: an observational nationwide study. J Thromb Haemost. 2015;13:31-40.

\section{Tables}

Due to technical limitations, tables are only available as a download in the Supplemental Files section.

\section{Supplementary Files}

This is a list of supplementary files associated with this preprint. Click to download.

- Table1st.xlsx 